\title{
LOCAL COHOMOLOGY OF BINOMIAL EDGE IDEALS AND THEIR GENERIC INITIAL IDEALS
}

\author{
JOSEP ÀLVAREZ MONTANER
}

\begin{abstract}
We provide a Hochster type formula for the local cohomology modules of binomial edge ideals. As a consequence we obtain a simple criterion for the Cohen-Macaulayness of these ideals and we describe their Castelnuovo-Mumford regularity and their Hilbert series. We also prove a conjecture of Conca, De Negri and Gorla relating the graded components of the local cohomology modules of binomial edge ideals and their generic initial ideals.
\end{abstract}

\section{INTRODUCTION}

Binomial edge ideals have been introduced by Herzog, Hibi, Hreinsdottir, Kahle, and Rauh in [18] and independently by Ohtani in [29] as a way to associate a binomial ideal to a given simple graph $G$ on the vertex set $[n]=\{1, \ldots, n\}$. Binomial edge ideals can be also understood as a natural generalization of the ideal of 2 -minors of a $2 \times n$-generic matrix. Over the last few years there has been a flurry of activity in the Combinatorial Commutative Algebra community trying to translate algebraic properties of the ideals to combinatorial properties of the graph following the same spirit as in the research done in the case of monomial edge ideals. Indeed, there has been a lot of effort in capturing the Cohen-Macaulay property of these ideals [4, 15, 18, 30, 31, 32], to study the Betti numbers and the Castelnuovo-Mumford regularity [14, 16, 19, 21, 22, 26, 24, 25, 33, 37] or the Hilbert series [3, 23, 28, 34]. We should point out that most of the results mentioned here only work for specific classes of graphs such as closed graphs, block graphs, or bipartite graphs among others.

An essential tool that has been used to address the same questions for monomial edge ideals is the celebrated Hochster's formula which originally appeared in [20] and one may consult different versions in the books [35], [6] and [27]. Hochster's formula provide a decomposition of the local cohomology modules $H_{\mathfrak{m}}^{r}(A / I)$ of the Stanley-Reisner ring $A / I$ associated to a squarefree monomial ideal $I \subseteq A$, where $A=\mathbb{K}\left[x_{1}, \ldots, x_{n}\right]$ is a polynomial ring with coefficients in a field $\mathbb{K}$. In an equivalent incarnation, Hochster's formula describes the Hilbert series of $A / I$. The lack of such a formula for binomial edge ideals is one of the reasons that the questions mentioned above have been so challenging.

In a different line of research, Conca, De Negri and Gorla [8, 9, 10, 11] introduced a family of ideals inspired by work of Cartwright and Sturmfels in [7] which, roughly speaking, are $\mathbb{Z}^{n}$-graded ideals whose multigraded generic initial ideal is radical. They proved in [11] that

Partially supported by Generalitat de Catalunya 2017 SGR-932 project and Spanish Ministerio de Economía y Competitividad MTM2015-69135-P. the author is a member of the Barcelona Graduate School of Mathematics (BGSMath). 
binomial edge ideals are Cartwright-Sturmfels ideals computing explicitly the corresponding generic initial ideal. When comparing invariants of Cartwright-Sturmfels ideals with those of its generic initial ideals they came up with the following:

Conjecture 1.1. [11, Conjecture 1.14] Let $I \subseteq A$ be a Cartwright-Sturmfels ideal and gin $(I)$ its $\mathbb{Z}^{n}$-graded generic initial ideal. Then one has:

$$
\operatorname{dim}_{\mathbb{K}} H_{\mathfrak{m}}^{r}(A / I)_{a}=\operatorname{dim}_{\mathbb{K}} H_{\mathfrak{m}}^{r}(A / \operatorname{gin}(I))_{a}
$$

for every $r \in \mathbb{N}$ and every $a \in \mathbb{Z}^{n}$.

Indeed, this conjecture implies the equality of the extremal Betti numbers of $I$ and gin $(I)$, their projective dimension and their Castelnuovo-Mumford regularity as well (see also [11, Conjecture 1.13]). A more general version of this conjecture has been proved recently by Conca and Varbaro in [13].

The aim of this work is twofold, on one hand we want to provide a Hochster type formula for the local cohomology modules of binomial edge ideals using the theory of local cohomology spectral sequences developed by the author, Boix and Zarzuela in [2]. On the other hand we want to prove Conjecture 1.1 for binomial edge ideals using a completely different approach to the one considered in [13]. The advantage of our methods is that we provide a very explicit description of the graded pieces of these local cohomology modules. On the contrary, our techniques are very specific for this case of binomial edge ideals so we cannot expect to recover the general statement proved in [13].

The structure of the paper is as follows. In Section 2 we present the basics on local cohomology spectral sequences developed in [2]. Their description are technically involved so we will try to avoid some of the details since, in the case that some extra assumptions on the ideal are satisfied, the spectral sequences become enormously simplified. This is the case that we will use throughout this work. The version that we are going to consider is presented in Theorem 2.2 and, as a consequence of the degeneration of this spectral sequence, we have a very general version of Hochster's formula (see Theorem 2.3).

In Section 3 we find the main results of this work. After some work needed to verify that binomial edge ideals satisfy those extra assumptions, we obtain the main result of this work in Theorem 3.9 which is a Hochster type formula for the local cohomology modules of a binomial edge ideal. As an immediate consequence we obtain in Corollary 3.11 a simple criterion to decide the Cohen-Macaulayness of binomial edge ideals. Moreover, we also obtain a formula for the $\mathbb{Z}$-graded Hilbert series of these local cohomology modules in Theorem 3.12.

In Section 4 we turn our attention to the local cohomology modules of the generic initial ideal of a binomial edge ideal. Our main result is Theorem 4.5 where we settle Conjecture 1.1. Our approach is to use a coarser version of the original Hochster formula for this very particular monomial ideal (see Theorem 4.4) that has the same structure as the one obtained in Theorem 3.9. In this way we reduce the problem to a simple comparision of the graded pieces of the building blocks of these decompositions. Finally we present a formula for the Castelnuovo-Mumford regularity of binomial edge ideals in Theorem 4.6 and a formula for the $\mathbb{Z}^{n}$-graded Hilbert series in Theorem 4.7. 
Aknowledgements: We greatly appreciate Aldo Conca, Emmanuela de Negri and Elisa Gorla for bringing to our attention their work on Cartwright-Sturmfels ideals and, in particular, Conjecture 1.1 during a workshop in honor of Peter Schenzel held in Osnabrück. We also want to thank Alberto F. Boix and Santiago Zarzuela for so many discussions about local cohomology spectral sequences we had over the last few years.

\section{Local COHOMOLOGY SPECTRAL SEQUENCE}

Let $A$ be a commutative Noetherian ring containing a field $\mathbb{K}$ and let $(\mathcal{P}, \preccurlyeq)$ be a partially ordered set of ideals $I_{p} \subseteq A$ ordered by reverse inclusion. Namely, given $p, q \in \mathcal{P}$ we have $p \preccurlyeq q$ if and only if $I_{p} \supseteq I_{q}$. We will assume that there exists a maximal ideal $\mathfrak{m}$ of $A$ containing all the ideals in the poset. In the sequel, if no confusion arise, we will indistinctly refer to the ideal $I_{p} \in \mathcal{P}$ or the element $p \in \mathcal{P}$ for simplicity.

A formalism to produce local cohomology spectral sequences associated to a poset was given in [2]. The construction of these spectral sequences is technically involved so we are just going to sketch the main ideas behind its construction and refer to [2] for details. Associated to the poset $\mathcal{P}$ we consider the inverse system of $A$-modules $A /[*]:=\left(A / I_{p}\right)_{p \in \mathcal{P}}$. Applying the $\mathfrak{m}$-torsion functor $\Gamma_{\mathfrak{m}}$ to this inverse system we obtain another inverse system $\Gamma_{\mathfrak{m}}(A /[*]):=\left(\Gamma_{\mathfrak{m}}\left(A / I_{p}\right)\right)_{p \in \mathcal{P}}$. The category of inverse systems of $A$-modules over a poset has enough injectives so we may consider the right derived inverse systems $\mathbb{R}^{j} \Gamma_{\mathfrak{m}}(A /[*])$. The spectral sequence given in [2, Theorem 5.6] involves the right derived functors of the limit of this inverse system. In the form we are interested in this work it reads as:

$$
E_{2}^{i, j}=\mathbb{R}^{i} \lim _{p \in \mathcal{P}} \mathbb{R}^{j} \Gamma_{\mathfrak{m}}(A /[*]) \Longrightarrow H_{\mathfrak{m}}^{i+j}\left(\lim _{p \in \mathcal{P}} A / I_{p}\right) .
$$

Notice that the spectral sequence starts with an inverse system of $A$-modules but converges to the usual local cohomology module which lives in the category of $A$-modules.

We point out that we have a lot of flexibility on the poset $\mathcal{P}$ that we are considering and we will make a strong use of this fact. Indeed, associated to an ideal $I \subseteq A$ admitting a decomposition $I=I_{1} \cap \ldots \cap I_{n}$ for certain ideals $I_{1}, \ldots, I_{n}$ of $A$, it is natural to consider the poset $\mathcal{P}_{I}$ given by all the possible sums of the ideals in the decomposition. So, any ideal $I_{p} \in \mathcal{P}_{I}$ is just a certain sum of ideals among $I_{1}, \ldots, I_{n}$. This is the approach considered in [2] (see also [1]) when dealing with monomial ideals or arrangements of linear subspaces. For the case of binomial edge ideals we are going to consider a poset $\mathcal{Q}_{I}$ which is a refinement of $\mathcal{P}_{I}$ that adjusts much better to our purposes (see Definition 3.3). We should mention that the inverse systems $\left(A / I_{p}\right)_{p \in \mathcal{P}_{I}}$ and $\left(A / I_{q}\right)_{q \in \mathcal{Q}_{I}}$ are cofinal so there will be no problem when computing their limit (see Remark 3.8).

At first sight the spectral sequence seems quite complicated but it becomes enormously simplified in the case that we consider some extra assumptions on the poset $\mathcal{P}$ associated to the ideal $I$. 
Key assumptions:

(A1) $\mathcal{P}$ is a subset of a distributive lattice of ideals of $A$.

(A2) $A / I_{p}$ is Cohen-Macaulay for all $p \in \mathcal{P}$.

(A3) $I_{q}$ is not contained in any minimal prime of $I_{p}$ for any $p \npreceq q$.

Assumption (A1) implies that $\lim _{p \in \mathcal{P}} A / I_{p}=A / I$ (see [2, Proposition 5.1]). On the other hand, Assumptions (A2) and (A3) ensure that the spectral sequence degenerates at the $E_{2^{-}}$ page (see [2, Theorem 5.22]). Actually, (A2) and (A3) are satisfied whenever $A / I_{p}$ are CohenMacaulay domains for all $p \in \mathcal{P}$.

In order to check Assumption (A2) it will be useful to consider the following result, due to Bouchiba and Kabbaj [5, Theorem 2.1], on the Cohen-Macaulayness of the tensor product (see also [17] and [36]).

Theorem 2.1. [5, Theorem 2.1] Let $A$ and $B$ be $\mathbb{K}$-algebras such that $A \otimes_{\mathbb{K}} B$ is Noetherian. Then, the following conditions are equivalent:

i) $A$ and $B$ are Cohen-Macaulay.

ii) $A \otimes_{\mathbb{K}} B$ is Cohen-Macaulay.

Let $1_{\mathcal{P}}$ be a terminal element that we add to the poset $\mathcal{P}$. To any $p \in \mathcal{P}$ we may consider the order complex associated to the subposet $\left(p, 1_{\mathcal{P}}\right):=\left\{z \in \mathcal{P} \mid \quad p<z<1_{\mathcal{P}}\right\}$ and its reduced simplicial cohomology groups $\widetilde{H}^{i}\left(\left(p, 1_{\mathcal{P}}\right) ; \mathbb{K}\right)$. Under Assuptions (A1), (A2) and (A3), the spectral sequence for local cohomology modules reads as follows:

Theorem 2.2. [2, Theorem 5.22] Let $A$ be a commutative Noetherian ring containing a field $\mathbb{K}$ and let $\mathcal{P}$ be a poset associated to an ideal $I \subseteq A$ satisfying Assuptions (A1), (A2) and (A3). Then, there exists a first quadrant spectral sequence of the form:

$$
E_{2}^{i, j}=\bigoplus_{j=d_{p}} H_{\mathfrak{m}}^{d_{p}}\left(A / I_{p}\right)^{\oplus M_{i, p}} \Longrightarrow H_{\mathfrak{m}}^{i+j}(A / I)
$$

where $M_{i, p}=\operatorname{dim}_{\mathbb{K}} \widetilde{H}^{i-d_{p}-1}\left(\left(p, 1_{\mathcal{P}}\right) ; \mathbb{K}\right)$ and $d_{p}=\operatorname{dim} A / I_{p}$. Moreover, this spectral sequence degenerates at the $E_{2}$-page.

From the degeneration of the spectral sequence we get that, for each $0 \leq r \leq \operatorname{dim}(A)$, there is an increasing, finite filtration $\left\{H_{i}^{r}\right\}_{0 \leq i \leq b}$ of $H_{\mathfrak{m}}^{r}(A / I)$ by $A$-modules, or equivalently we have a collection of short exact sequences

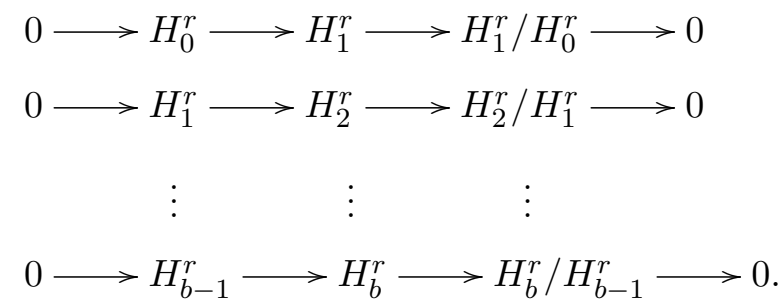


where $H_{b}^{r}=H_{\mathfrak{m}}^{r}(A / I)$ for some $b \in \mathbb{N}$ and, for each $r$ the quotients $H_{i}^{r} / H_{i-1}^{r}$ decompose in the following manner:

$$
H_{i}^{r} / H_{i-1}^{r} \cong \bigoplus_{\left\{p \in \mathcal{P} \mid r-i=d_{p}\right\}} H_{\mathfrak{m}}^{d_{p}}\left(A / I_{p}\right)^{\oplus M_{i, p}}
$$

These short exact sequences split as $\mathbb{K}$-vector spaces so we obtain the following generalization of the celebrated Hochster's formula.

Theorem 2.3. Let $A$ be a commutative Noetherian ring containing a field $\mathbb{K}$ and let $\mathcal{P}$ be a poset associated to an ideal $I \subseteq$ A satisfying Assuptions (A1), (A2) and (A3). Then, there is a $\mathbb{K}$-vector space isomorphism

$$
H_{\mathfrak{m}}^{r}(A / I) \cong \bigoplus_{p \in \mathcal{P}} H_{\mathfrak{m}}^{d_{p}}\left(A / I_{p}\right)^{\oplus M_{r, p}}
$$

It is proved in [2, Sections 5.3 and 7.1] that we may add an enhanced structure to the spectral sequence considered in Theorem 2.2. In our case we want to consider a g-graded structure where $\mathfrak{g}$ is either $\mathbb{Z}$ or $\mathbb{Z}^{n}$. Then, the graded version of Theorem 2.3 reads as

$$
H_{\mathfrak{m}}^{r}(A / I)_{a} \cong \bigoplus_{p \in \mathcal{P}} H_{\mathfrak{m}}^{d_{p}}\left(A / I_{p}\right)_{a}^{\oplus M_{r, p}}
$$

for every $a \in \mathfrak{g}$.

The original Hochster formula is nothing but a very particular case of Theorem 2.3.

Corollary 2.4. Let $A=\mathbb{K}\left[x_{1}, \ldots, x_{n}\right]$ be a polynomial ring with coefficients over a field $\mathbb{K}$ and let $I \subseteq A$ be a squarefree monomial ideal. Let $\mathcal{P}_{I}$ be the poset given by all the possible sums of the ideals in the minimal primary decomposition of $I$. Then, there is a $\mathbb{K}$-vector space isomorphism

$$
H_{\mathfrak{m}}^{r}(A / I) \cong \bigoplus_{p \in \mathcal{P}} H_{\mathfrak{m}}^{d_{p}}\left(A / I_{p}\right)^{\oplus M_{r, p}}
$$

Moreover we have a decomposition as graded $\mathbb{K}$-vector spaces.

\section{LOCAL COHOMOLOGY MOdUleS OF BinOmial EDGE IDEALS}

Let $A=\mathbb{K}\left[x_{1}, \ldots, x_{n}, y_{1}, \ldots, y_{n}\right]$ be a polynomial ring with coefficients over a field $\mathbb{K}$ and let $G$ be a graph on the vertex set $[n]=\{1, \ldots, n\}$. The binomial edge ideal associated to $G$ is the ideal

$$
\left.J_{G}=\left\langle\Delta_{i j}\right|\{i, j\} \text { is an edge of } G\right\rangle,
$$

where $\Delta_{i j}=x_{i} y_{j}-x_{j} y_{i}$ denote the corresponding 2 -minor of a $2 \times n$ matrix

$$
\left(\begin{array}{llll}
x_{1} & x_{2} & \cdots & x_{n} \\
y_{1} & y_{2} & \cdots & y_{n}
\end{array}\right)
$$

With the $\mathbb{Z}^{n}$-graded structure on $A$ given by $\operatorname{deg}\left(x_{i}\right)=\operatorname{deg}\left(y_{i}\right)=e_{i} \in \mathbb{Z}^{n}$, where $e_{i}$ is the $i$-th unit vector, we have that $J_{G}$ is a $\mathbb{Z}^{n}$-graded ideal. We may also associate the usual $\mathbb{Z}$-graded structure given by $\operatorname{deg}\left(x_{i}\right)=\operatorname{deg}\left(y_{i}\right)=1$. 
Binomial edge ideals are radical ideals whose primary decomposition is nicely described. Namely, given $S \subseteq[n]$, let $c=c(S)$ be the number of connected components of $G \backslash S$, that we denote by $G_{1}, \ldots, G_{c}$, and let $\widetilde{G}_{i}$ be the complete graph on the vertices of $G_{i}$. We set

$$
P_{S}(G)=\left\langle x_{i}, y_{i} \mid i \in S\right\rangle+J_{\widetilde{G}_{1}}+\cdots+J_{\widetilde{G}_{c}}
$$

and let $|S|$ be the number of elements of $S$.

Theorem 3.1 ([18]). Let $G$ be a graph on $[n]$, and $J_{G}$ its binomial edge ideal in $A$. Then we have:

i) $P_{S}(G)$ is a prime ideal of height $n-c+|S|$ for every $S \subseteq[n]$.

ii) We have a decomposition

$$
J_{G}=\bigcap_{S \subseteq[n]} P_{S}(G) .
$$

iii) $P_{S}(G)$ is a minimal prime of $J_{G}$ if and only if either $S=\emptyset$ or $S \neq \emptyset$ and, for each $i \in S$, we have $c(S \backslash\{i\})<c(S)$.

iv) If $G$ is a connected graph on $[n]$, then $P_{\emptyset}(G)$ is a minimal prime of $J_{G}$.

3.1. A poset associated to a binomial edge ideal. Let $J_{G}$ be the binomial edge ideal associated to a graph $G$ on the set of vertices $[n]$. Associated to the minimal primary decomposition

$$
J_{G}=P_{S_{1}}(G) \cap \cdots \cap P_{S_{r}}(G)
$$

we may consider the poset $\mathcal{P}_{J_{G}}$ given by all the possible sums of the ideals in the decomposition ordered by reverse inclusion. An interesting feature that occurs in the case of monomial ideals or arrangement of linear subspaces is that the corresponding poset only contains prime ideals. This is no longer true for the case of binomial edge ideals as the following example shows.

Example 3.2. Let $\mathbb{K}\left[x_{1}, \ldots, x_{5}, y_{1}, \ldots, y_{5}\right]$ be a polynomial ring with coefficients over a field $\mathbb{K}$ and let $J_{G}$ be the binomial edge ideal associated to the 5 -path $G:=\{\{1,2\},\{2,3\},\{3,4\},\{4,5\}\}$. Its minimal primary decomposition is $J_{G}=I_{1} \cap I_{2} \cap I_{3} \cap I_{4} \cap I_{5}$ where:

$$
\begin{aligned}
& I_{1}:=P_{\emptyset}(G)=\left\langle\Delta_{12}, \Delta_{13}, \Delta_{14}, \Delta_{15}, \Delta_{23}, \Delta_{24}, \Delta_{25}, \Delta_{34}, \Delta_{35}, \Delta_{45}\right\rangle \\
& I_{2}:=P_{\{2\}}(G)=\left\langle x_{2}, y_{2}, \Delta_{34}, \Delta_{35}, \Delta_{45}\right\rangle \\
& I_{3}:=P_{\{3\}}(G)=\left\langle x_{3}, y_{3}, \Delta_{12}, \Delta_{45}\right\rangle \\
& I_{4}:=P_{\{4\}}(G)=\left\langle x_{4}, y_{4}, \Delta_{12}, \Delta_{13}, \Delta_{23}\right\rangle \\
& I_{5}:=P_{\{2,4\}}(G)=\left\langle x_{2}, y_{2}, x_{4}, y_{4}\right\rangle
\end{aligned}
$$

We have that $I_{2}+I_{4}+I_{5}=\left\langle x_{2}, y_{2}, x_{4}, y_{4}, \Delta_{13}, \Delta_{35}\right\rangle$ admits a primary decomposition of the form

$$
I_{2}+I_{4}+I_{5}=\left\langle x_{2}, y_{2}, x_{4}, y_{4}, \Delta_{13}, \Delta_{15}, \Delta_{35}\right\rangle \cap\left\langle x_{2}, y_{2}, x_{3}, y_{3}, x_{4}, y_{4}\right\rangle .
$$

We point out that these prime components as well as its sum $\left\langle x_{2}, y_{2}, x_{3}, y_{3}, x_{4}, y_{4}, \Delta_{15}\right\rangle$ are indeed elements of the poset $\mathcal{P}_{J_{G}}$. The complete list of ideals in the poset $\mathcal{P}_{J_{G}}$ contains $I_{1}, I_{2}, I_{3}, I_{4}, I_{5}$ and $\left\langle x_{2}, y_{2}, \Delta_{13}, \Delta_{14}, \Delta_{15}, \Delta_{34}, \Delta_{35}, \Delta_{45}\right\rangle,\left\langle x_{3}, y_{3}, \Delta_{12}, \Delta_{14}, \Delta_{15}, \Delta_{24}, \Delta_{25}, \Delta_{45}\right\rangle$, 
$\left\langle x_{2}, y_{2}, x_{3}, y_{3}, \Delta_{45}\right\rangle,\left\langle x_{2}, y_{2}, x_{4}, y_{4}, \Delta_{13}\right\rangle,\left\langle x_{4}, y_{4}, \Delta_{12}, \Delta_{13}, \Delta_{15}, \Delta_{23}, \Delta_{25}, \Delta_{35}\right\rangle,\left\langle x_{2}, y_{2}, x_{4}, y_{4}, \Delta_{35}\right\rangle$, $\left\langle x_{3}, y_{3}, x_{4}, y_{4}, \Delta_{12}\right\rangle,\left\langle x_{2}, y_{2}, x_{4}, y_{4}, \Delta_{13}, \Delta_{35}\right\rangle,\left\langle x_{2}, y_{2}, x_{3}, y_{3}, x_{4}, y_{4}\right\rangle,\left\langle x_{2}, y_{2}, x_{3}, y_{3}, \Delta_{14}, \Delta_{15}, \Delta_{45}\right\rangle$, $\left\langle x_{2}, y_{2}, x_{4}, y_{4}, \Delta_{13}, \Delta_{15}, \Delta_{35}\right\rangle,\left\langle x_{3}, y_{3}, x_{4}, y_{4}, \Delta_{12}, \Delta_{15}, \Delta_{25}\right\rangle,\left\langle x_{2}, y_{2}, x_{3}, y_{3}, x_{4}, y_{4}, \Delta_{15}\right\rangle$.

All the ideals in the poset, except for the one presented in blue, are prime ideals of the form $P_{S}(H)$ for some graph $H$ in the vertex set $[n]$ and some $S \subseteq[n]$.

The fact that some elements of the poset $\mathcal{P}_{J_{G}}$ may not be prime ideals is not a big issue in the computation of the corresponding local cohomology modules. Indeed, the building blocks $H_{\mathfrak{m}}^{d_{p}}\left(A / I_{p}\right)$ associated to non prime ideals appearing in the formula given in Theorem 2.3 admit some further decomposition applying Theorem 2.3 as many times as necessary. Is for this reason that it seems more natural to associate the following poset to the minimal primary decomposition of the ideal $J_{G}$ containing only prime ideals and being cofinal with $\mathcal{P}_{J_{G}}$.

Definition 3.3. Let $J_{G}$ be the binomial edge ideal associated to a graph $G$ on the set of vertices $[n]$. We construct iteratively a poset $\mathcal{Q}_{J_{G}}$ associated to $J_{G}$ as follows: The ideals contained in $\mathcal{Q}_{J_{G}}$ are the prime ideals in $\mathcal{P}_{J_{G}}$, the prime ideals in the posets $\mathcal{P}_{I_{q}}$ of sums of the ideals in the decomposition of every non prime ideal $I_{q}$ in $\mathcal{P}_{J_{G}}$ and the prime ideals that we obtain repeating this procedure every time we find a non prime ideal. This process stops after a finite number of steps since we only have a finite number of prime ideals of the form $P_{S}(H)$ for some graph $H$ on the set of vertices $[n]$ and some $S \subseteq[n]$.

Remark 3.4. In Example 3.2, we have that the poset $\mathcal{Q}_{J_{G}}$ contains all the ideals in $\mathcal{P}_{J_{G}}$ except for $\left\langle x_{2}, y_{2}, x_{4}, y_{4}, \Delta_{13}, \Delta_{35}\right\rangle$.

Before going on, we will fix some notation that will be useful in the rest of this work.

Notation 3.5. Let $I_{q}$ be a prime ideal in the poset $\mathcal{Q}_{J_{G}}$. Assume that it has the form

$$
I_{q}=P_{S}(H)=\left\langle x_{i}, y_{i} \mid i \in S\right\rangle+J_{\widetilde{H}_{1}}+\cdots+J_{\widetilde{H}_{c_{q}}},
$$

where $H$ is a graph in the set of vertices $[n], S \subseteq[n], H_{1}, \ldots, H_{c_{q}}$ are the connected components of $H \backslash S$ and $\widetilde{H}_{i}$ denotes the complete graph on the vertices of $H_{i}$. Let $n_{i, q}$ be the number of vertices of $H_{i}$, then we may rename and reorder the variables $x_{i}, y_{i}$ in such a way that

$$
\left\langle x_{i}, y_{i} \mid i \in S\right\rangle \subseteq A_{0}:=\mathbb{K}\left[x_{j}, y_{j} \mid j \in S\right]=\mathbb{K}\left[x_{1}^{0}, \ldots, x_{|S|}^{0}, y_{1}^{0}, \ldots, y_{|S|}^{0}\right]
$$

and, for $i=1, \ldots, c_{q}$

$$
J_{\widetilde{H}_{i}} \subseteq A_{i}:=\mathbb{K}\left[x_{j}, y_{j} \mid j \in H_{i}\right]=\mathbb{K}\left[x_{1}^{i}, \ldots, x_{n_{i, q}}^{i}, y_{1}^{i}, \ldots, y_{n_{i, q}}^{i}\right] .
$$

In particular, we have

$$
\begin{gathered}
A=A_{0} \otimes_{\mathbb{K}} A_{1} \otimes_{\mathbb{K}} \cdots \otimes_{\mathbb{K}} A_{c_{q}} \\
A / I_{q}=A_{1} / J_{\widetilde{H}_{1}} \otimes_{\mathbb{K}} \cdots \otimes_{\mathbb{K}} A_{c_{q}} / J_{\widetilde{H}_{c_{q}}} .
\end{gathered}
$$

Notice that $d_{q}:=\operatorname{dim} A / I_{q}=n-|S|+c=d_{1}+\cdots+d_{c_{q}}$, where $d_{i}:=\operatorname{dim} A_{i} / J_{\widetilde{H}_{i}}=n_{i, q}+1$. In the sequel we will denote by $\mathfrak{m}_{i}$ the homogeneous maximal ideal of $A_{i}$. 
3.2. Local cohomology of binomial edge ideals. In order to apply Theorem 2.3 for binomial edge ideals we have to make sure that the poset $\mathcal{Q}_{J_{G}}$ that we constructed in Definition 3.3 satisfies Assumptions (A1), (A2) and (A3).

Recall that the ideal of $2 \times 2$-minors of a $2 \times n$-matrix is Cohen-Macaulay and thus $A / I_{q}$ is also Cohen-Macaulay by Theorem 2.1. Therefore Assumptions (A2) and (A3) are satisfied for the poset $\mathcal{Q}_{J_{G}}$ since it only contains Cohen-Macaulay prime ideals. In order to prove Assumption (A1) we will first do the following

Discussion 3.6. Let $I_{q_{1}}$ and $I_{q_{2}}$ be a prime ideals in the poset $\mathcal{Q}_{J_{G}}$ of the form

$$
\begin{gathered}
I_{q_{1}}=P_{S_{1}}\left(H_{1}\right)=\left\langle x_{i}, y_{i} \mid i \in S_{1}\right\rangle+J_{\widetilde{H}_{1,1}}+\cdots+J_{\widetilde{H}_{1, c_{q_{1}}}}, \\
I_{q_{2}}=P_{S_{2}}\left(H_{2}\right)=\left\langle x_{j}, y_{j} \mid j \in S_{2}\right\rangle+J_{\widetilde{H}_{2,1}}+\cdots+J_{\widetilde{H}_{2, c_{2}}},
\end{gathered}
$$

for some graphs $H_{1}$ and $H_{2}$ in the set of vertices $[n]$. In order to study the intersection $I_{q_{1}} \cap I_{q_{2}}$ we notice that both ideals are sums of ideals in different sets of separated variables and thus we may reduce the problem to the study of the intersection of either two prime monomial ideals, a prime monomial ideal and a binomial edge ideal of a complete graph or the intersection of two binomial edge ideal of complete graphs. In these cases we have the following description of the generators in terms of the corresponding graphs:

- A minimal set of generators of $\left\langle x_{i}, y_{i} \mid i \in S_{1}\right\rangle \cap\left\langle x_{i}, y_{i} \mid i \in S_{2}\right\rangle$ only contains elements

- $x_{i}$ and $y_{i}$ for any common vertex $i \in S_{1} \cap S_{2}$.

- $x_{i} x_{j}, x_{i} y_{j}, y_{i} x_{j}$ and $y_{i} y_{j}$ for any pair of vertices $i \in S_{1} \backslash S_{2}$ and $j \in S_{2} \backslash S_{1}$.

- A minimal set of generators of $\left\langle x_{i}, y_{i} \mid i \in S_{1}\right\rangle \cap J_{\widetilde{H}_{2, t}}$ only contains elements

- $\Delta_{i j}$ whenever the edge $\{i, j\}$ belongs to the complete graph $\widetilde{H}_{2, t}$ and either $i$ or $j$ is a vertex of $S_{1}$.

- $x_{i} \Delta_{k \ell}$ and $y_{i} \Delta_{k \ell}$ whenever $i \in S_{1}$ and the edge $\{k, \ell\}$ belongs to $\widetilde{H}_{2, t}$ but $k, \ell \notin S_{1}$.

Analogously for $\left\langle x_{i}, y_{i} \mid i \in S_{2}\right\rangle \cap J_{\widetilde{H}_{1, s}}$.

- A minimal set of generators of $J_{\widetilde{H}_{1, s}} \cap J_{\widetilde{H}_{2, t}}$ only contains elements

- $\Delta_{i j}$ for common edges $\{i, j\}$.

- $\Delta_{i j} \Delta_{k \ell}$ for edges $\{i, j\}$ in $\widetilde{H}_{1, s}$ and $\{k, \ell\}$ in $\widetilde{H}_{2, t}$ sharing at most one vertex.

- $x_{j} y_{\ell} \Delta_{i k}-x_{\ell} y_{k} \Delta_{i j}=x_{i} y_{k} \Delta_{j \ell}-x_{j} y_{i} \Delta_{k \ell}$ for edges $\{i, j\}$ in $\widetilde{H}_{1, s},\{k, \ell\}$ in $\widetilde{H}_{2, t}$ and $\{j, k\}$ being a common edge.

Assumption (A1) follows from the following

Proposition 3.7. Let $J_{G}$ be the binomial edge ideal associated to a graph $G$ on the set of vertices $[n]$. Then, the poset $\mathcal{Q}_{J_{G}}$ is a subset of a distributive lattice.

Proof. Given prime ideals $I_{q_{1}}, I_{q_{2}}, I_{q_{3}}$ in the poset $\mathcal{Q}_{J_{G}}$ we have to check

$$
\left(I_{q_{1}}+I_{q_{2}}\right) \cap I_{q_{3}}=\left(I_{q_{1}} \cap I_{q_{3}}\right)+\left(I_{q_{2}} \cap I_{q_{3}}\right) .
$$


The inclusion $\supseteq$ always holds so let's prove the other inclusion. Assume that

$$
\begin{gathered}
I_{q_{1}}=P_{S_{1}}\left(H_{1}\right)=\left\langle x_{i}, y_{i} \mid i \in S_{1}\right\rangle+J_{\widetilde{H}_{1,1}}+\cdots+J_{\widetilde{H}_{1, c_{q_{1}}}}, \\
I_{q_{2}}=P_{S_{2}}\left(H_{2}\right)=\left\langle x_{j}, y_{j} \mid j \in S_{2}\right\rangle+J_{\widetilde{H}_{2,1}}+\cdots+J_{\widetilde{H}_{2, q_{2}}}, \\
I_{q_{3}}=P_{S_{3}}\left(H_{3}\right)=\left\langle x_{j}, y_{j} \mid j \in S_{3}\right\rangle+J_{\widetilde{H}_{3,1}}+\cdots+J_{\widetilde{H}_{3, c_{q_{3}}}} .
\end{gathered}
$$

Recall that $I_{q_{1}}+I_{q_{2}}$ is not necessarily a prime ideal. Indeed, we have a presentation

$$
I_{q_{1}}+I_{q_{2}}=\left\langle x_{i}, y_{i} \mid i \in S_{1} \cup S_{2}\right\rangle+J_{K_{1}}+\cdots+J_{K_{c}},
$$

where $K_{t}$ are non necessarily complete graphs corresponding to the connected components of $K \backslash S_{1} \cup S_{2}$ with $K$ being a graph in $[n]$. A minimal set of generators of $\left(I_{q_{1}}+I_{q_{2}}\right) \cap I_{q_{3}}$ is contained in the intersection of the prime ideal $\left\langle x_{i}, y_{i} \mid i \in S_{1} \cup S_{2}\right\rangle+J_{\widetilde{K}_{1}}+\cdots+J_{\widetilde{K}_{c}}$ with $I_{q_{3}}$. In particular these elements have the form considered in Discussion 3.6.

Now, let $u \in\left(I_{q_{1}}+I_{q_{2}}\right) \cap I_{q_{3}}$ be an element in a minimal set of generators.

- If $u=x_{i}$ or $y_{i}$, then $i \in S_{1} \cup S_{2}$ and $i \in S_{3}$ and thus $u \in\left(I_{q_{1}} \cap I_{q_{3}}\right)+\left(I_{q_{2}} \cap I_{q_{3}}\right)$.

- If $u$ is $x_{i} x_{j}, x_{i} y_{j}, y_{i} x_{j}$ or $y_{i} y_{j}$ then $i \in S_{1} \cup S_{2} \backslash S_{3}$ and $j \in S_{3} \backslash S_{1} \cup S_{2}$ and thus $u \in\left(I_{q_{1}} \cap I_{q_{3}}\right)+\left(I_{q_{2}} \cap I_{q_{3}}\right)$.

- If $u=\Delta_{i j}$ it could be the case that the edge $\{i, j\}$ belongs to $K_{t}$, and consequently is an edge of $H_{1}$ or $H_{2}$, and either $i$ or $j$ is a vertex of $S_{3}$. It could also be the way around, $\{i, j\}$ belongs to $H_{3}$ and either $i$ or $j$ is a vertex of $S_{1} \cup S_{2}$. It may also happen that $\{i, j\}$ is a common edge of $K_{t}$ and $H_{3}$. In any case we have $u \in\left(I_{q_{1}} \cap I_{q_{3}}\right)+\left(I_{q_{2}} \cap I_{q_{3}}\right)$.

- If $u$ is $x_{i} \Delta_{k \ell}$ or $y_{i} \Delta_{k \ell}$ then $i \in S_{1} \cup S_{2}$ and the edge $\{k, \ell\}$ belongs to $H_{3}$ but $k, \ell \notin$ $S_{1} \cup S_{2}$ or the way around, $i \in S_{3}$ and the edge $\{k, \ell\}$ belongs to $K_{t}$ but $k, \ell \notin S_{3}$. We have $u \in\left(I_{q_{1}} \cap I_{q_{3}}\right)+\left(I_{q_{2}} \cap I_{q_{3}}\right)$.

- If $u=\Delta_{i j} \Delta_{k \ell}$ then the edges $\{i, j\}$ in $K_{t}$ and $\{k, \ell\}$ in $H_{3}$ share at most one vertex. Since $\{i, j\}$ belongs to $H_{1}$ or $H_{2}$, we have $u \in\left(I_{q_{1}} \cap I_{q_{3}}\right)+\left(I_{q_{2}} \cap I_{q_{3}}\right)$.

- If $u=x_{j} y_{\ell} \Delta_{i k}-x_{\ell} y_{k} \Delta_{i j}=x_{i} y_{k} \Delta_{j \ell}-x_{j} y_{i} \Delta_{k \ell}$ then $\{i, j\}$ is an edge in $K_{t}$ and consequently an edge of $H_{1}$ or $H_{2},\{k, \ell\}$ in $H_{3}$ and $\{j, k\}$ is a common edge. We have $u \in\left(I_{q_{1}} \cap I_{q_{3}}\right)+\left(I_{q_{2}} \cap I_{q_{3}}\right)$.

Remark 3.8. The prime ideals in the minimal primary decomposition of the binomial edge ideal $J_{G}$ are the maximal elements of the posets $\mathcal{Q}_{J_{G}}$ and $\mathcal{P}_{J_{G}}$. One deduces that the inverse systems $\left(A / I_{q}\right)_{q \in \mathcal{Q}_{J_{G}}}$ and $\left(A / I_{q}\right)_{q \in \mathcal{Q}_{J_{G}}}$ are cofinal. In particular

$$
\lim _{q \in \mathcal{Q}_{J_{G}}} A / I_{q}=\lim _{p \in \mathcal{P}_{J_{G}}} A / I_{p}
$$

This limit is isomorphic to $A / I$ under the conditions of Assumption (A1).

Now we are ready to present the main result of this work which is a Hochster's type decomposition for the local cohomology modules associated to a binomial edge ideal. 
Theorem 3.9. Let $A=\mathbb{K}\left[x_{1}, \ldots, x_{n}, y_{1}, \ldots, y_{n}\right]$ be a polynomial ring with coefficients over a field $\mathbb{K}$ and $\mathfrak{m}$ be its homogeneous maximal ideal. Let $J_{G} \subseteq A$ be the binomial edge ideal associated to a graph $G$ in the set of vertices $[n]$. Let $\mathcal{Q}_{J_{G}}$ be the poset associated to a minimal primary decomposition of $J_{G}$. Then, the local cohomology modules with respect to $\mathfrak{m}$ of $A / J_{G}$ admit the following decomposition as $\mathbb{K}$-vector spaces:

$$
H_{\mathfrak{m}}^{r}\left(A / J_{G}\right) \cong \bigoplus_{q \in \mathcal{Q}_{J_{G}}} H_{\mathfrak{m}}^{d_{q}}\left(A / I_{q}\right)^{\oplus M_{r, q}}
$$

where $M_{r, q}=\operatorname{dim}_{\mathbb{K}} \widetilde{H}^{r-d_{q}-1}\left(\left(q, 1_{\mathcal{Q}_{J_{G}}}\right) ; \mathbb{K}\right)$. Moreover we have a decomposition as graded $\mathbb{K}$ vector spaces.

Example 3.10. Let $A=\mathbb{K}\left[x_{1}, \ldots, x_{8}, y_{1}, \ldots, y_{8}\right]$ be a polynomial ring with coefficients over a field $\mathbb{K}$ and let $J_{G} \subseteq A$ be the binomial edge ideal associated to the complete bipartite graph $G=K_{3,5}$. Its minimal primary decomposition is $J_{G}=I_{p_{1}} \cap I_{p_{2}} \cap I_{p_{3}}$ where

$$
\begin{aligned}
& I_{p_{1}}=\left\langle x_{1}, x_{2}, x_{3}, x_{4}, x_{5}, y_{1}, y_{2}, y_{3}, y_{4}, y_{5}\right\rangle \\
& I_{p_{2}}=\left\langle\Delta_{12}, \ldots, \Delta_{78}\right\rangle \\
& I_{p_{3}}=\left\langle x_{6}, x_{7}, x_{8}, y_{6}, y_{7}, y_{8}\right\rangle
\end{aligned}
$$

The associated poset $\mathcal{Q}_{J_{G}}$ has the form

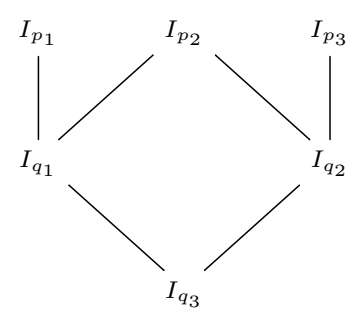

where

$$
\begin{aligned}
& I_{q_{1}}=\left\langle x_{1}, x_{2}, x_{3}, x_{4}, x_{5}, y_{1}, y_{2}, y_{3}, y_{4}, y_{5}, \Delta_{67}, \Delta_{68}, \Delta_{78}\right\rangle \\
& I_{q_{2}}=\left\langle x_{6}, x_{7}, x_{8}, y_{6}, y_{7}, y_{8}, \Delta_{12}, \Delta_{13}, \Delta_{14}, \Delta_{15}, \Delta_{23}, \Delta_{24}, \Delta_{25}, \Delta_{34}, \Delta_{35}, \Delta_{45}\right\rangle \\
& I_{q_{3}}=\left\langle x_{1}, x_{2}, x_{3}, x_{4}, x_{5}, x_{6}, x_{7}, x_{8}, y_{1}, y_{2}, y_{3}, y_{4}, y_{5}, y_{6}, y_{7}, y_{8}\right\rangle
\end{aligned}
$$

Then:

\begin{tabular}{|c|c|c|c|c|}
\hline$q$ & $\left(q, 1_{\mathcal{Q}_{J_{G}}}\right)$ & $\operatorname{dim}_{k} \tilde{H}^{-1}$ & $\operatorname{dim}_{k} \tilde{H}^{0}$ & $\operatorname{dim}_{k} \tilde{H}^{1}$ \\
\hline$p_{1}$ & $\emptyset$ & 1 & - & - \\
$p_{2}$ & $\emptyset$ & 1 & - & - \\
$p_{3}$ & $\emptyset$ & 1 & - & - \\
$q_{1}$ & $\bullet$ & - & 1 & - \\
$q_{2}$ & $\bullet$ & - & 1 & - \\
$q_{3}$ & $\vee \bigvee$ & - & - & - \\
\hline
\end{tabular}

It follows from Theorem 3.9 that 


$$
\begin{aligned}
& H_{\mathfrak{m}}^{5}\left(A / J_{G}\right)=H_{\mathfrak{m}}^{4}\left(A / I_{q_{1}}\right), \quad H_{\mathfrak{m}}^{6}\left(A / J_{G}\right)=H_{\mathfrak{m}}^{6}\left(A / I_{p_{1}}\right), \quad H_{\mathfrak{m}}^{7}\left(A / J_{G}\right)=H_{\mathfrak{m}}^{6}\left(A / I_{q_{2}}\right), \\
& H_{\mathfrak{m}}^{9}\left(A / J_{G}\right)=H_{\mathfrak{m}}^{9}\left(A / I_{p_{2}}\right), \quad H_{\mathfrak{m}}^{10}\left(A / J_{G}\right)=H_{\mathfrak{m}}^{10}\left(A / I_{p_{3}}\right)
\end{aligned}
$$

In particular, there are five local cohomology modules different from zero so $A / J_{G}$ is not Cohen-Macaulay.

From the vanishing of local cohomology modules we may deduce a simple criterion for the Cohen-Macaulayness of a binomial edge ideal in terms of the associated poset.

Corollary 3.11. Let $A=\mathbb{K}\left[x_{1}, \ldots, x_{n}, y_{1}, \ldots, y_{n}\right]$ be a polynomial ring with coefficients over a field $\mathbb{K}$ and $\mathfrak{m}$ be its homogeneous maximal ideal. Let $J_{G} \subseteq A$ be the binomial edge ideal associated to a graph $G$ in the set of vertices $[n]$. Let $\mathcal{Q}_{J_{G}}$ be the poset associated to a minimal primary decomposition of $J_{G}$. Then the following are equivalent:

i) $J_{G}$ is Cohen-Macaulay.

ii) $M_{r, q}=\operatorname{dim}_{\mathbb{K}} \widetilde{H}^{r-d_{q}-1}\left(\left(q, 1_{\mathcal{Q}_{J_{G}}}\right) ; \mathbb{K}\right)=0$ for all $r \neq \operatorname{dim} A / J_{G}$ and all $q \in \mathcal{Q}_{J_{G}}$.

Using Theorem 3.9 and some results obtained by Conca and Herzog in [12] we are able to provide a formula for the $\mathbb{Z}$-graded Hilbert series.

Let $H S(M ; t):=\sum_{a \in \mathbb{Z}} \operatorname{dim}_{\mathbb{K}}\left(M_{a}\right) t^{a}$ be the $\mathbb{Z}$-graded Hilbert series of a $\mathbb{Z}$-graded module $M$ such that $\operatorname{dim}_{\mathbb{K}}\left(M_{a}\right)<+\infty$ for all $a \in \mathbb{Z}$. In the case that $M$ is the local cohomology of a binomial edge ideal we have:

Theorem 3.12. Let $A=\mathbb{K}\left[x_{1}, \ldots, x_{n}, y_{1}, \ldots, y_{n}\right]$ be a polynomial ring with coefficients over a field $\mathbb{K}$ and $\mathfrak{m}$ be its homogeneous maximal ideal. Let $J_{G} \subseteq A$ be the binomial edge ideal associated to a graph $G$ in the set of vertices $[n]$. Let $\mathcal{Q}_{J_{G}}$ be the poset associated to a minimal primary decomposition of $J_{G}$. Then

$$
\begin{aligned}
H S\left(H_{\mathfrak{m}}^{r}\left(A / J_{G}\right) ; t\right) & =\sum_{\substack{q \in \mathcal{Q}_{J_{G}} \\
I_{q}}} M_{r, q} \frac{t^{-d_{q}}}{\left(1-t^{-1}\right)^{d_{q}}} \\
& +\sum_{\substack{q \in \mathcal{Q}_{J_{G}} \\
I_{q} \text { not monomial }}} M_{r, q} \frac{\prod_{i=1}^{c_{q}}\left(\left(n_{i, q}-1\right) t^{-n_{i, q}}+t^{-\left(n_{i, q}+1\right)}\right)}{\left(1-t^{-1}\right)^{d_{q}}}
\end{aligned}
$$

where $M_{r, q}=\operatorname{dim}_{\mathbb{K}} \widetilde{H}^{r-d_{q}-1}\left(\left(q, 1_{\mathcal{Q}_{J_{G}}}\right) ; \mathbb{K}\right)$.

Proof. From the decomposition given in Theorem 3.9 and the additivity of the Hilbert series with respect to short exact sequences we have

$$
H S\left(H_{\mathfrak{m}}^{r}\left(A / J_{G}\right) ; t\right)=\sum_{q \in \mathcal{Q}_{J_{G}}} M_{r, q} H S\left(H_{\mathfrak{m}}^{d_{q}}\left(A / I_{q}\right) ; t\right)
$$


If $I_{q}=\left\langle x_{i}, y_{i} \mid i \in S\right\rangle$ is a monomial ideal, then, using Hochster's formula we have

$$
H S\left(H_{\mathfrak{m}}^{d_{q}}\left(A / I_{q}\right) ; t\right)=\left(\frac{t^{-1}}{1-t^{-1}}\right)^{d_{q}} .
$$

If $I_{q}$ is not a monomial ideal, we assume that $A / I_{q}=A_{1} / J_{\widetilde{H}_{1}} \otimes_{\mathbb{K}} \cdots \otimes_{\mathbb{K}} A_{c} / J_{\widetilde{H}_{c_{q}}}$. Given the relation given by the Hilbert series of a Cohen-Macaulay ring and its canonical module we get

$$
\begin{aligned}
H S\left(\omega_{A / I_{q}} ; t\right) & =(-1)^{d_{q}} H S\left(A / I_{q} ; t^{-1}\right)=(-1)^{d_{q}} \prod_{i=1}^{c_{q}} H S\left(A_{i} / J_{\widetilde{H}_{i}} ; t^{-1}\right) \\
& =(-1)^{d_{q}} \prod_{i=1}^{c_{q}}(-1)^{d_{i}} H S\left(\omega_{A_{i} / J_{\widetilde{H}_{i}}} ; t\right)=\prod_{i=1}^{c_{q}} H S\left(\omega_{A_{i} / J_{\widetilde{H}_{i}}} ; t\right) \\
& =\prod_{i=1}^{c_{q}} \frac{t^{n_{i, q}}\left(\left(n_{i, q}-1\right)+t\right)}{(1-t)^{d_{i}}}
\end{aligned}
$$

where the last assertion follows from [12]. Then, taking into account that graded local duality reverses the degrees, we get

$$
H S\left(H_{\mathfrak{m}}^{d_{q}}\left(A / I_{q}\right) ; t\right)=\prod_{i=1}^{c_{q}} H S\left(H_{\mathfrak{m}_{\mathfrak{i}}}^{d_{i}}\left(A_{i} / J_{\widetilde{H}_{i}}\right) ; t\right)=\prod_{i=1}^{c_{q}} \frac{\left(t^{-1}\right)^{n_{i, q}}\left(\left(n_{i, q}-1\right)+t^{-1}\right)}{\left(1-t^{-1}\right)^{d_{i}}}
$$

and the result follows.

Example 3.13. Let $A=\mathbb{K}\left[x_{1}, \ldots, x_{8}, y_{1}, \ldots, y_{8}\right]$ be a polynomial ring with coefficients over a field $\mathbb{K}$ and let $J_{G} \subseteq A$ be the binomial edge ideal associated to the complete bipartite graph $G=K_{3,5}$. Then

$$
\begin{aligned}
& H S\left(H_{\mathfrak{m}}^{5}\left(A / J_{G}\right) ; t\right)=H S\left(H_{\mathfrak{m}}^{4}\left(A / I_{q_{1}}\right) ; t\right)=\frac{2 t^{-3}+t^{-4}}{\left(1-t^{-1}\right)^{4}}, \\
& H S\left(H_{\mathfrak{m}}^{6}\left(A / J_{G}\right) ; t\right)=H S\left(H_{\mathfrak{m}}^{6}\left(A / I_{p_{1}}\right) ; t\right)=\frac{t^{-6}}{\left(1-t^{-1}\right)^{6}}, \\
& H S\left(H_{\mathfrak{m}}^{7}\left(A / J_{G}\right) ; t\right)=H S\left(H_{\mathfrak{m}}^{6}\left(A / I_{q_{2}}\right) ; t\right)=\frac{4 t^{-5}+t^{-6}}{\left(1-t^{-1}\right)^{6}}, \\
& H S\left(H_{\mathfrak{m}}^{9}\left(A / J_{G}\right) ; t\right)=H S\left(H_{\mathfrak{m}}^{9}\left(A / I_{p_{2}}\right) ; t\right)=\frac{7 t^{-8}+t^{-9}}{\left(1-t^{-1}\right)^{9}}, \\
& H S\left(H_{\mathfrak{m}}^{10}\left(A / J_{G}\right) ; t\right)=H S\left(H_{\mathfrak{m}}^{10}\left(A / I_{p_{3}}\right) ; t\right)=\frac{t^{-10}}{\left(1-t^{-1}\right)^{10}} .
\end{aligned}
$$

4. LocAl COHOMOlogy MODUles OF GENERIC INITIAL IDEALS OF BINOMIAL EDGE IDEALS

Let $J_{G} \subseteq \mathbb{K}\left[x_{1}, \ldots, x_{n}, y_{1}, \ldots, y_{n}\right]$ be the binomial edge ideal associated to a graph $G$ on $[n]$. A precise description of the corresponding generic initial ideal gin $\left(J_{G}\right)$ has been given by 
Conca, De Negri and Gorla in [11] in order to prove that binomial edge ideals belong to the class of Cartwright-Sturmfels ideals introduced in [7]. Namely, we have:

Theorem 4.1. [11, Theorem 2.1] Let $J_{G}$ be the binomial edge ideal associated to a graph $G$ on $[n]$. Then, the $\mathbb{Z}^{n}$-graded generic initial ideal of $J_{G}$ is

$$
\left.\operatorname{gin}\left(J_{G}\right):=\left\langle x_{i} x_{j} y_{a_{1}} \cdots y_{a_{v}}\right|\left\{i, a_{1}, \cdots, a_{v}, j\right\} \text { is a path in } G\right\rangle \text {. }
$$

An important feature of generic initial ideals is that they behave well with respect to minimal primary decompositions.

Proposition 4.2. [11, Corollary 1.12] Let $J_{G}=P_{S_{1}}(G) \cap \cdots \cap P_{S_{r}}(G)$ be the minimal primary decomposition of the binomial edge ideal $J_{G}$ associated to a graph $G$ on $[n]$. Then we have a decomposition

$$
\operatorname{gin}\left(J_{G}\right)=\operatorname{gin}\left(P_{S_{1}}(G)\right) \cap \cdots \cap \operatorname{gin}\left(P_{S_{r}}(G)\right)
$$

If we take a close look, we will see that the decomposition of $\operatorname{gin}\left(J_{G}\right)$ that we obtain is not necessarily a minimal primary decomposition.

Proposition 4.3. Let $G$ be a graph on $[n]$, and $S \subseteq[n]$. Then:

i) $\operatorname{gin}\left(P_{S}(G)\right)=\left\langle x_{i}, y_{i} \mid i \in S\right\rangle+\operatorname{gin}\left(J_{\widetilde{G}_{1}}\right)+\cdots+\operatorname{gin}\left(J_{\widetilde{G}_{c}}\right)$. In particular, it is a squarefree monomial ideal of height $n-c+|S|$ for every $S \subseteq[n]$.

ii) If $\widetilde{G}$ is a complete graph on the vertices $\{1, \ldots, t\} \subseteq[n]$, then

$$
\operatorname{gin}\left(J_{\widetilde{G}}\right)=\left\langle x_{i} x_{j} \mid 1 \leq i<j \leq t\right\rangle=\bigcap_{i=1}^{t}\left\langle x_{1}, \ldots \widehat{x}_{i}, \ldots, x_{t}\right\rangle
$$

which is a Cohen-Macaulay monomial ideal.

4.1. A poset associated to the generic initial ideal of a binomial edge ideal. Let $G$ be a graph on the set of vertices $[n]$ and let $J_{G}$ be its associated binomial edge ideal. Its generic ideal $\operatorname{gin}\left(J_{G}\right)$ is monomial so we may use the original Hochster's formula to describe its local cohomology modules. However, since our goal is to compare the local cohomology modules of both ideals, we are going to use Theorem 2.2 considering a poset associated to $\operatorname{gin}\left(J_{G}\right)$ that will be more useful for our purposes.

Let $J_{G}=P_{S_{1}}(G) \cap \cdots \cap P_{S_{r}}(G)$ be the minimal primary decomposition of the binomial edge ideal $J_{G}$. By Proposition 4.2 we have the decomposition

$$
\operatorname{gin}\left(J_{G}\right)=\operatorname{gin}\left(P_{S_{1}}(G)\right) \cap \cdots \cap \operatorname{gin}\left(P_{S_{r}}(G)\right) .
$$

Associated to this decomposition we are going to consider the poset $\mathcal{Q}_{\operatorname{gin}\left(J_{G}\right)}$ which contains $\operatorname{gin}\left(I_{q}\right)$ for all ideals $I_{q}$ in $\mathcal{Q}_{J_{G}}$. It is clear that $\mathcal{Q}_{\operatorname{gin}\left(J_{G}\right)}$ is the same poset as $\mathcal{Q}_{J_{G}}$ where we simply have a different label for the ideals so, if no confusion arise, we will simple denote both as $\mathcal{Q}_{J_{G}}$. In this way we emphasize that the decomposition obtained in Theorem 3.9 and Theorem 4.4 for the local cohomology of $J_{G}$ and $\operatorname{gin}\left(J_{G}\right)$ respectively, are the same since the order complexes $\left(q, 1_{\mathcal{Q}_{J_{G}}}\right)$ are exactly the same in both cases and thus they have the same reduced cohomology. 
4.2. Local cohomology of the generic initial ideal of a binomial edge ideal. In this Section we present a decomposition of the local cohomology modules of the generic initial ideal of a binomial edge ideal which is coarser than the original Hochster's formula for monomial ideals but it will be more appropriate for our purposes. In order to apply Theorem 2.3 we have to check first that the poset $\mathcal{Q}_{J_{G}}$ associated to the generic initial ideal of a binomial edge ideal satisfies Assumptions (A1), (A2) and (A3).

In this context we are dealing with squarefree monomial ideals so $\mathcal{Q}_{J_{G}}$ is a subset of a distributive lattice of ideals of $A$ so Assumption (A1) is satisfied. Indeed

$$
\lim _{q \in \mathcal{Q}_{J_{G}}} A / \operatorname{gin}\left(I_{q}\right) \cong A / \operatorname{gin}\left(J_{G}\right)
$$

since $\mathcal{Q}_{J_{G}}$ is cofinal with the poset $\mathcal{P}_{\operatorname{gin}\left(J_{G}\right)}$ associated to a minimal primary decomposition of $\operatorname{gin}\left(J_{G}\right)$. We have

$$
A / \operatorname{gin}\left(I_{q}\right)=A_{1} / \operatorname{gin}\left(J_{\widetilde{H}_{1}}\right) \otimes_{\mathbb{K}} \cdots \otimes_{\mathbb{K}} A_{c} / \operatorname{gin}\left(J_{\widetilde{H}_{c}}\right),
$$

so it is Cohen-Macaulay by Proposition 4.3 and Theorem 2.1 so Assumption (A2) is also satisfied.

In order to prove Assumption (A3) we only have to notice that the ideals $I_{p}$ are binomial edge ideals of some graphs, say $G_{p}$, whose connected components are complete graphs. Therefore, the condition $I_{p} \subseteq I_{q}$ is equivalent to the reverse inclusion $G_{p} \supseteq G_{q}$, which is also equivalent to $\operatorname{gin}\left(I_{p}\right) \subseteq \operatorname{gin}\left(I_{q}\right)$.

The coarser version of Hochster's formula that we obtain is the following:

Theorem 4.4. Let $A=\mathbb{K}\left[x_{1}, \ldots, x_{n}, y_{1}, \ldots, y_{n}\right]$ be a polynomial ring with coefficients over a field $\mathbb{K}$ and $\mathfrak{m}$ be its homogeneous maximal ideal. Let $J_{G} \subseteq A$ be the binomial edge ideal associated to a graph $G$ in the set of vertices $[n]$. Let $\mathcal{Q}_{J_{G}}$ be the poset associated to a minimal primary decomposition of $J_{G}$. Then the local cohomology modules with respect to $\mathfrak{m}$ of $A / \operatorname{gin}\left(J_{G}\right)$ admit the following decomposition as $\mathbb{K}$-vector spaces:

$$
H_{\mathfrak{m}}^{r}\left(A / \operatorname{gin}\left(J_{G}\right)\right) \cong \bigoplus_{p \in \mathcal{Q}_{J_{G}}} H_{\mathfrak{m}}^{d_{p}}\left(A / \operatorname{gin}\left(I_{p}\right)\right)^{\oplus M_{r, p}}
$$

where $M_{i, p}=\operatorname{dim}_{\mathbb{K}} \widetilde{H}^{i-d_{p}-1}\left(\left(p, 1_{\mathcal{Q}_{J_{G}}}\right) ; \mathbb{K}\right)$. Moreover this is a decomposition of graded $\mathbb{K}$-vector spaces.

As a consequence of the decomposition results for local cohomology modules obtained in Theorem 3.9 and Theorem 4.4, we can give an affirmative answer to Conjecture 1.1 for the case of binomial edge ideals.

Theorem 4.5. Let $A=\mathbb{K}\left[x_{1}, \ldots, x_{n}, y_{1}, \ldots, y_{n}\right]$ be a polynomial ring with coefficients over a field $\mathbb{K}$ and $\mathfrak{m}$ be its homogeneous maximal ideal. Let $J_{G} \subseteq A$ be the binomial edge ideal associated to a graph $G$ in the set of vertices $[n]$. Then we have

$$
\operatorname{dim}_{\mathbb{K}} H_{\mathfrak{m}}^{r}\left(A / J_{G}\right)_{a}=\operatorname{dim}_{\mathbb{K}} H_{\mathfrak{m}}^{r}\left(A / \operatorname{gin}\left(J_{G}\right)\right)_{a}
$$

for every $r \in \mathbb{N}$ and every $a \in \mathbb{Z}^{n}$. 
Proof. By Theorem 3.9 and Theorem 4.4 we only have to check that

$$
\operatorname{dim}_{\mathbb{K}} H_{\mathfrak{m}}^{d_{q}}\left(A / I_{q}\right)_{a}=\operatorname{dim}_{\mathbb{K}} H_{\mathfrak{m}}^{d_{q}}\left(A / \operatorname{gin}\left(I_{q}\right)\right)_{a}
$$

for every $a \in \mathbb{Z}^{n}$ and every $q \in \mathcal{Q}_{J_{G}}$. Assuming that the ideal $I_{q}$ has the form

$$
I_{q}=P_{S}(H)=\left\langle x_{i}, y_{i} \mid i \in S\right\rangle+J_{\widetilde{H}_{1}}+\cdots+J_{\widetilde{H}_{c}}
$$

it is then enough to prove that

$$
\left.\operatorname{dim}_{\mathbb{K}} H_{\mathfrak{m}_{i}}^{d_{p}}\left(A_{i} / J_{\widetilde{H}_{i}}\right)_{a}=\operatorname{dim}_{\mathbb{K}} H_{\mathfrak{m}_{i}}^{d_{p}}\left(A_{i} / \operatorname{gin}\left(J_{\widetilde{H_{i}}}\right)\right)\right)_{a}
$$

for every $a \in \mathbb{Z}^{n}$ where $\widetilde{H_{i}}$ is a complete graph that, without loss of generality, we consider in the set of vertices $\left\{1, \ldots, n_{i, q}\right\} \subseteq[n]$ and thus

$$
\operatorname{gin}\left(J_{\widetilde{H}_{i}}\right)=\left\langle x_{i} x_{j} \mid 1 \leq i<j \leq n_{i, q}\right\rangle=\bigcap_{j=1}^{n_{i, q}}\left\langle x_{1}, \ldots \widehat{x_{j}}, \ldots, x_{n_{i, q}}\right\rangle .
$$

In general we have $\left.\operatorname{dim}_{\mathbb{K}} H_{\mathfrak{m}_{i}}^{d_{p}}\left(A_{i} / J_{\widetilde{H}_{i}}\right)_{a} \leq \operatorname{dim}_{\mathbb{K}} H_{\mathfrak{m}_{i}}^{d_{p}}\left(A_{i} / \operatorname{gin}\left(J_{\widetilde{H}_{i}}\right)\right)\right)_{a}$ so the result follows if

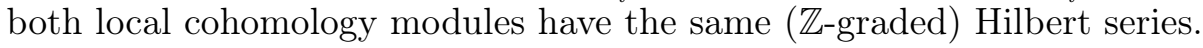

The $f$-vector of the simplicial complex associated to the squarefree monomial ideal $\operatorname{gin}\left(J_{\widetilde{H}_{i}}\right)$ is $\left(f_{-1}, f_{0}\right)=\left(1, n_{i, q}\right)$. Therefore

$$
H S\left(A_{i} / \operatorname{gin}\left(J_{\widetilde{H}_{i}}\right) ; t\right)=\frac{\left(n_{i, q}-1\right) t+1}{(1-t)^{d_{i}}}=(-1)^{d_{i}} H S\left(\omega_{A_{i} / J_{\widetilde{H}_{i}}} ; t^{-1}\right)
$$

and, by graded local duality,

$$
H S\left(H_{\mathfrak{m}_{\mathfrak{i}}}^{d_{i}}\left(A_{i} / J_{\widetilde{H}_{i}}\right) ; t\right)=\frac{t^{-n_{i, q}}\left(\left(n_{i, q}-1\right)+t^{-1}\right)}{\left(1-t^{-1}\right)^{d_{i}}} .
$$

Comparing with the Hilbert series obtained in the proof of Theorem 3.12 the result follows.

As a consequence of this result we have that the extremal Betti numbers, the projective dimension and the Castelnuovo-Mumford regularity of $J_{G}$ and $\operatorname{gin}\left(J_{G}\right)$ are equal. In particular we have:

Proposition 4.6. Let $A=\mathbb{K}\left[x_{1}, \ldots, x_{n}, y_{1}, \ldots, y_{n}\right]$ be a polynomial ring with coefficients over a field $\mathbb{K}$ and $\mathfrak{m}$ be its homogeneous maximal ideal. Let $J_{G} \subseteq A$ be the binomial edge ideal associated to a graph $G$ in the set of vertices $[n]$. Let $\mathcal{Q}_{J_{G}}$ be the poset associated to a minimal primary decomposition of $J_{G}$. Then,

$$
\operatorname{reg}\left(A / J_{G}\right)=\max _{r \geq 0, q \in \mathcal{Q}_{J_{G}}}\left\{r-d_{q} \mid M_{r, p} \neq 0\right\},
$$

where $M_{r, q}=\operatorname{dim}_{\mathbb{K}} \widetilde{H}^{r-d_{q}-1}\left(\left(q, 1_{\mathcal{Q}_{J_{G}}}\right) ; \mathbb{K}\right)$.

Proof. We have

$\operatorname{reg}\left(A / J_{G}\right)=\max _{r \geq 0}\left\{\operatorname{end}\left(H_{\mathfrak{m}}^{r}\left(A / J_{G}\right)\right)+r\right\}=\max _{r \geq 0, q \in \mathcal{Q}_{J_{G}}}\left\{\right.$ end $\left.\left(H_{\mathfrak{m}}^{d_{q}}\left(A / I_{q}\right)\right)+r \mid \quad M_{r, p} \neq 0\right\}$. 
Recall that $\operatorname{gin}\left(J_{\widetilde{H}_{i}}\right)=\left\langle x_{i} x_{j} \mid 1 \leq i<j \leq n_{i, q}\right\rangle$ and thus, from a direct computation, we get end $\left(H_{\mathfrak{m}_{\mathfrak{i}}}^{d_{i}}\left(A_{i} / \operatorname{gin}\left(J_{\widetilde{H}_{i}}\right)\right)\right)=-d_{i}$. Therefore

$$
\text { end }\left(H_{\mathfrak{m}}^{d_{q}}\left(A / I_{q}\right)\right)=\operatorname{end}\left(H_{\mathfrak{m}}^{d_{q}}\left(A / \operatorname{gin}\left(I_{q}\right)\right)\right)=-d_{q}
$$

and the result follows.

Finally we turn our attention to the $\mathbb{Z}^{n}$-graded Hilbert series

$$
H S\left(M ; t_{1}, \ldots, t_{n}\right):=\sum_{a \in \mathbb{Z}^{n}} \operatorname{dim}_{\mathbb{K}}\left(M_{a}\right) t_{1}^{a_{1}} \cdots t_{n}^{a_{n}}
$$

where $a=\left(a_{1}, \ldots, a_{n}\right) \in \mathbb{Z}^{n}$. The formula that we obtain is a little bit involved. To start with, for each ideal $I_{q} \in \mathcal{Q}_{J_{G}}$, we will rename and reorder the variables $t_{i}$ accordingly so we have

$$
\left\{t_{1}, \ldots, t_{n}\right\}=\left\{t_{1}^{0}, \ldots, t_{|S|}^{0}, t_{1}^{1}, \ldots, t_{n_{1, q}}^{1}, \ldots, t_{1}^{c_{q}}, \ldots, t_{n_{c_{q}, q}}^{c_{q}}\right\} .
$$

In the case that $I_{q}=\left\langle x_{i}, y_{i} \mid i \in S\right\rangle$ is a monomial ideal, we rename and reorder the variables as

$$
\left\{t_{1}, \ldots, t_{n}\right\}=\left\{t_{1, q}, \ldots, t_{|S|, q}, t_{|S|+1, q}, \ldots, t_{n, q}\right\} .
$$

Theorem 4.7. Let $A=\mathbb{K}\left[x_{1}, \ldots, x_{n}, y_{1}, \ldots, y_{n}\right]$ be a polynomial ring with coefficients over a field $\mathbb{K}$ and $\mathfrak{m}$ be its homogeneous maximal ideal. Let $J_{G} \subseteq A$ be the binomial edge ideal associated to a graph $G$ in the set of vertices $[n]$. Let $\mathcal{Q}_{J_{G}}$ be the poset associated to a minimal primary decomposition of $J_{G}$. Then

$$
\begin{aligned}
H S\left(H_{\mathfrak{m}}^{r}\left(A / J_{G}\right) ; t_{1}, \ldots, t_{n}\right) & =\sum_{\substack{q \in \mathcal{Q}_{J_{G}} \\
I_{q}}} M_{r, q} \prod_{i=|S|+1}^{n} \frac{\left(t_{i, q}\right)^{-2}}{\left(1-\left(t_{i, q}\right)^{-1}\right)^{2}} \\
& +\sum_{\substack{q \in \mathcal{Q}_{J_{G}} \\
I_{q} \text { not monomial }}} M_{r, q} \prod_{i=1}^{c_{q}} \frac{\left(t_{1}^{i}\right)^{-1} \cdots\left(t_{n_{i, q}}^{i}\right)^{-1}}{\left(1-\left(t_{1}^{i}\right)^{-1}\right) \cdots\left(1-\left(t_{n_{i, q}}^{i}\right)^{-1}\right)}\left(\left(n_{i, q}-1\right)+\sum_{j=1}^{n_{i, q}} \frac{\left(t_{j}^{i}\right)^{-1}}{\left(1-\left(t_{j}^{i}\right)^{-1}\right)}\right)
\end{aligned}
$$

where $M_{r, q}=\operatorname{dim}_{\mathbb{K}} \widetilde{H}^{r-d_{q}-1}\left(\left(q, 1_{\mathcal{Q}_{J_{G}}}\right) ; \mathbb{K}\right)$.

Proof. From the decomposition given in Theorem 3.9 and the additivity of Hilbert series on short exact sequences we have

$$
H S\left(H_{\mathfrak{m}}^{r}\left(A / J_{G}\right) ; t_{1}, \ldots, t_{n}\right)=\sum_{q \in \mathcal{Q}_{J_{G}}} M_{r, q} H S\left(H_{\mathfrak{m}}^{d_{q}}\left(A / I_{q}\right) ; t_{1}, \ldots, t_{n}\right)
$$

In the case that $I_{q}=\left\langle x_{i}, y_{i} \mid i \in S\right\rangle$ is a monomial ideal we have

$$
H S\left(H_{\mathfrak{m}}^{d_{q}}\left(A / I_{q}\right) ; t_{1}, \ldots, t_{n}\right)=\prod_{i=|S|+1}^{n} \frac{\left(t_{i, q}\right)^{-2}}{\left(1-\left(t_{i, q}\right)^{-1}\right)^{2}}
$$


If $I_{q}$ is not a monomial ideal then

$$
\begin{gathered}
H S\left(H_{\mathfrak{m}}^{d_{q}}\left(A / I_{q}\right) ; t_{1}, \ldots, t_{n}\right)=\prod_{i=1}^{c_{q}} H S\left(H_{\mathfrak{m}_{i}}^{d_{i}}\left(A_{i} / J_{\widetilde{H}_{i}}\right) ; t_{1}^{i}, \ldots, t_{n_{i, q}}^{i}\right) \\
=\prod_{i=1}^{c_{q}} H S\left(H_{\mathfrak{m}_{i}}^{d_{i}}\left(A_{i} / \operatorname{gin}\left(J_{\widetilde{H}_{i}}\right)\right) ; t_{1}^{i}, \ldots, t_{n_{i, q}}^{i}\right) \\
=\prod_{i=1}^{c_{q}} \frac{\left(t_{1}^{i}\right)^{-1} \cdots\left(t_{n_{i, q}}^{i}\right)^{-1}}{\left(1-\left(t_{1}^{i}\right)^{-1}\right) \cdots\left(1-\left(t_{n_{i, q}}^{i}\right)^{-1}\right)}\left(\left(n_{i, q}-1\right)+\sum_{j=1}^{n_{i, q}} \frac{\left(t_{j}^{i}\right)^{-1}}{\left(1-\left(t_{j}^{i}\right)^{-1}\right)}\right)
\end{gathered}
$$

The last assertion follows from a direct computation using the usual Hochster's formula for the $\mathbb{Z}^{n}$-graded Hilbert series.

\section{REFERENCES}

[1] J. Àlvarez Montaner, R. García López, S. Zarzuela Armengou, Local cohomology, arrangements of subspaces and monomial ideals, Adv. in Math. 174 (2003), 35-56. 3

[2] J. Àlvarez Montaner, A. F. Boix and S. Zarzuela, On some local cohomology spectral sequences, Int. Math. Res. Not. (2018), https://doi.org/10.1093/imrn/rny186. 2, 3, 4, 5

[3] A. Banerjee and L. Núñez-Betancourt, Graph connectivity and binomial edge ideals, Proc. Amer. Math. Soc. 145 (2017), 487-499. 1

[4] D. Bolognini, A. Macchia and F. Strazzanti, Binomial edge ideals of bipartite graphs, European J. Combin. 70 (2018), 1-25. 1

[5] S. Bouchiba and S. Kabbaj, Tensor products of Cohen-Macaulay rings: solution to a problem of Grothendieck, J. Algebra 252 (2002), 65-73. 4

[6] W. Bruns and J. Herzog, Cohen-Macaulay rings, Cambridge Studies in Advanced Mathematics, 39. Cambridge University Press, Cambridge, 1993. 1

[7] D. Cartwright and B. Sturmfels, The Hilbert scheme of the diagonal in a product of projective spaces, Int. Math. Res. Not. 9 (2010), 1741-1771. 1, 13

[8] A. Conca, E. De Negri, E. Gorla, Universal Gröbner bases for maximal minors, Int. Math. Res. Not. 11 (2015), 3245-3262. 1

[9] A. Conca, E. De Negri and E. Gorla, Universal Gröbner bases and Cartwright-Sturmfels ideals, Int. Math. Res. Not. (2018), https://doi.org/10.1093/imrn/rny075. 1

[10] A. Conca, E. De Negri and E. Gorla, Multigraded generic initial ideals of determinantal ideals, in: Homological and Computational Methods in Commutative Algebra Springer INdAM Series 20, Springer 2017. 1

[11] A. Conca, E. De Negri and E. Gorla, Cartwright-Sturmfels ideals associated to graphs and linear spaces, J. Comb. Algebra 2 (2018), 231-257. 1, 2, 13

[12] A. Conca and J. Herzog, On the Hilbert function of determinantal rings and their canonical module, Proc. Amer. Math. Soc. 122 (1994), 677-681. 11, 12

[13] A. Conca and M. Varbaro, Square-free Groebner degenerations, preprint available at arXiv:1805.11923. 2

[14] H. de Alba and D. T. Hoang, On the extremal Betti numbers of the binomial edge ideal of closed graphs, Math. Nachr. 291 (2018), 28-40. 1

[15] V. Ene, J. Herzog and T. Hibi, Cohen-Macaulay binomial edge ideals, Nagoya Math. J. 204 (2011), 57-68. 1

[16] V. Ene and A. Zarojanu, On the regularity of binomial edge ideals, Math. Nachr. 288 (2015) 19-24. 1

[17] A. Grothendieck, Eléments de géométrie algébrique, Institut des Hautes Etudes Sci. Publ. Math., Vol. 24, Bures-sur-yvette, 1965. 4

[18] J. Herzog, T. Hibi, F. Hreinsdottir, T. Kahle and J. Rauh, Binomial edge ideals and conditional independence statements, Adv. in Applied Math. 45 (2010), 317-333. 1, 6 
[19] J. Herzog and G. Rinaldo, On the extremal Betti numbers of binomial edge ideals of block graphs, Electron. J. Combin. 25 (2018), no. 1, Paper 1.63. 1

[20] M. Hochster, Cohen-Macaulay rings, combinatorics, and simplicial complexes, Ring theory, II (Proc. Second Conf., Univ. Oklahoma, Norman, Okla., 1975), pp. 171-223. Lecture Notes in Pure and Appl. Math., Vol. 26, Dekker, New York, 1977. 1

[21] A. V. Jayanthan and A. Kumar, Regularity of binomial edge ideals of Cohen-Macaulay bipartite graphs, preprint available at arXiv:1806.02109. 1

[22] A. V. Jayanthan, N. Narayanan and B. V. Raghavendra Rao, Regularity of binomial edge ideals of certain block graphs, preprint available at arXiv:1601.01086. 1

[23] A. Kumar and R. Sarkar Hilbert series of binomial edge ideals, preprint available at arXiv:1807.07273. 1

[24] C. Mascia and G. Rinaldo, Krull dimension and regularity of binomial edge ideals of block graphs, preprint available at arXiv:1803.01239. 1

[25] C. Mascia and G. Rinaldo, Extremal Betti numbers of some Cohen-Macaulay binomial edge ideals, preprint available at arXiv:1809.03423. 1

[26] K. Matsuda and S. Murai, Regularity bounds for binomial edge ideals, J. Commut. Algebra 5 (2013) 141-149. 1

[27] E. Miller and B. Sturmfels, Combinatorial commutative algebra. Graduate Texts in Mathematics, 227. Springer-Verlag, New York, 2005. xiv +417 pp. 1

[28] F. Mohammadi and L. Sharifan, Hilbert function of binomial edge ideals, Comm. Algebra 42 (2014), 688-703. 1

[29] M. Ohtani, Graphs and ideals generated by some 2-minors, Comm. Alg. 39 (2011), 905-917. 1

[30] A. Rauf and G. Rinaldo, Construction of Cohen-Macaulay binomial edge ideals, Comm. Algebra., 42 (2014), 238-252. 1

[31] G. Rinaldo, Cohen-Macaulay binomial edge ideals of small deviation, Bull. Math. Soc. Sci. Math. Roumanie, 56(104) (2013), 497-503. 1

[32] G. Rinaldo, Cohen-Macaulay binomial edge ideals of cactus graphs, arXiv:1704.07106. 1

[33] S. Saeedi Madani and D. Kiani, Binomial edge ideals of graphs, Electron. J. Combin. 19 (2012) P44. 1

[34] P. Schenzel and S. Zafar, Algebraic properties of the binomial edge ideal of a complete bipartite graph, An. Stiint Univ. "Ovidius" Constanza Ser. Mat. 22 (2014), 217-237. 1

[35] R. P. Stanley, Combinatorics and commutative algebra, second edition, Progress in Mathematics, 41. Birkhäuser Boston, Inc., Boston, MA, 1996. 1

[36] K. I. Watanabe, T. Ishikawa, S. Tachibana and K. Otsuka, On tensor products of Gorenstein rings. J. Math. Kyoto Univ. 9 (1969) 413-423. 4

[37] Z. Zahid and S. Zafar, On the Betti numbers of some classes of binomial edge ideals, Electron. J. Combin. 20 (2013) P37. 1

Departament de Matemàtiques, Universitat Politècnica de Catalunya, Av. Diagonal 647, Barcelona 08028, Spain

E-mail address: Josep.Alvarez@upc.edu 